\title{
Small angular scale CMB anisotropies from CBI and BIMA experiments: Early universe or local structures?
}

\author{
Asantha Cooray* \\ Theoretical Astrophysics, California Institute of Technology, Pasadena, California 91125 \\ Alessandro Melchiorri ${ }^{\dagger}$ \\ Theoretical Astrophysics, University of Oxford, Oxford OX 3RH, England \\ (Received 20 April 2002; published 4 October 2002)
}

\begin{abstract}
The advent of high-resolution cosmic microwave background (CMB) experiments now allows studies on the temperature fluctuations at scales corresponding to a few arcminutes and below. Though the reported excess power at $l \sim 2000-6000$ by CBI and BIMA is roughly consistent with a secondary contribution resulting from the Sunyaev-Zeldovich effect, this requires a higher normalization for the matter power spectrum than measured by other means. In addition to a local redshift contribution, another strong possibility for anisotropies at very small scales involves nonstandard aspects of inflationary models. To distinguish between contributions from early universe and local structures, including a potential point source contribution, and to understand the extent to which structures at low redshifts contribute to small-scale temperature anisotropies, it may be necessary to perform a combined study involving $\mathrm{CMB}$ and the large-scale structure. We suggest a cross correlation of the temperature data with a map of the large-scale structure, such as the galaxy distribution. For next generation small angular scale CMB experiments, multifrequency observations may be a necessary aspect to allow an additional possibility to distinguish between these different scenarios.
\end{abstract}

DOI: 10.1103/PhysRevD.66.083001

PACS number(s): 98.70.Vc, 95.85.Nv, 98.80.Es

\section{INTRODUCTION}

The high-resolution, and high signal-to-noise, cosmic microwave background (CMB) experiments such as Boomerang [1], DASI [2], MAXIMA [3], and, more recently, VSA [4] have revolutionized the study of temperature anisotropies at arcminute scales. The multiple peaks observed by these experiments in the angular power spectrum of the CMB anisotropies have provided strong evidence for inflationary models with adiabatic initial conditions for structure formation.

The recent results from the CBI interferometer [5] confirm the presence of multiple peaks in the angular power spectrum with an unambiguous detection of the damping tail as expected. At smaller angular scales beyond the damping tail, however, the CBI experiment in the "deep field" configuration [6] and the BIMA array [7] have both reported the presence of an "excess" in temperature power at a level of about $\sim 500 \mu \mathrm{K}^{2}$ with a detection confidence at the level of $\sim 3 \sigma$ and above.

The presence of this excess is not consistent with the predicted damping of the primordial (primary) anisotropies [6] but can in principle be explained as due to a secondary effect $[8,9]$. At angular scales corresponding to projected galaxy clusters extents of the order of a few arcminutes and below, the Sunyaev-Zel'dovich effect (SZ) ([10]), in fact, produces a now well-known contribution. The SZ effect has now been directly imaged towards massive galaxy clusters [11], where the temperature of the scattering medium can reach as high

\footnotetext{
*Email address: asante@caltech.edu

${ }^{\dagger}$ Email address: melch@astro.ox.ac.uk
}

as $10 \mathrm{keV}$ producing temperature changes in the CMB of order $1 \mathrm{mK}$ at Rayleigh-Jeans (RJ) wavelengths and whose presence is a priori known based on optical data. These, and other unresolved clusters, contribute to the dominant anisotropy contribution at arcminute scales.

Even if the detection by the CBI and BIMA experiments marks an important milestone, however, it is still not clear if the excess can be easily explained by the SZ effect. There are several techniques, based on semianalytic computations and numerical simulations, that can be used to predict the SZ signal from a population of clusters. The most recent numerical simulations (see e.g. [12]), assuming cosmological parameters consistent with the primary anisotropies and other observables, predict, in general, an amplitude for the SZ power spectrum at small angular scales which is roughly an order of magnitude lower than that observed by two recent experiments.

As discussed in the literature [8,9], the key parameter in fixing the amplitude of the SZ signal is the value of the rms mass fluctuations on spheres of $8 \mathrm{Mpc} \mathrm{h}^{-1}\left(\sigma_{8}\right)$. Due to the highly nonlinear behavior, the SZ thermal contribution (see Fig. 2 left panel) is strongly dependent on $\sigma_{8}$ with $C_{l}^{\mathrm{SZ}}$ $\sim \sigma_{8}^{7}$. As we can see in Fig. 2, right panel, the power spectrum associated with the SZ effect (calculated following the techniques described in $[13,14]$ ) is in agreement with the new CBI and BIMA data only for values of $\sigma_{8} \geqslant 1$. This is consistent with the value of $\sigma_{8}=1.05 \pm 0.15$ at $95 \%$ C.L. recently found by Komatsu and Seljak [9] in a detailed analysis and with the numerical results obtained by both Bond et al. [8], White et al. [12], and in Ref. [15] $\left(\sigma_{8}\right.$ $=1.0-1.2$ ).

A value of $\sigma_{8} \geqslant 1$, while in agreement with cosmic shear data (see e.g. [16]) and the "old" cluster normalization [17], is in disagreement with new cluster abundance results 

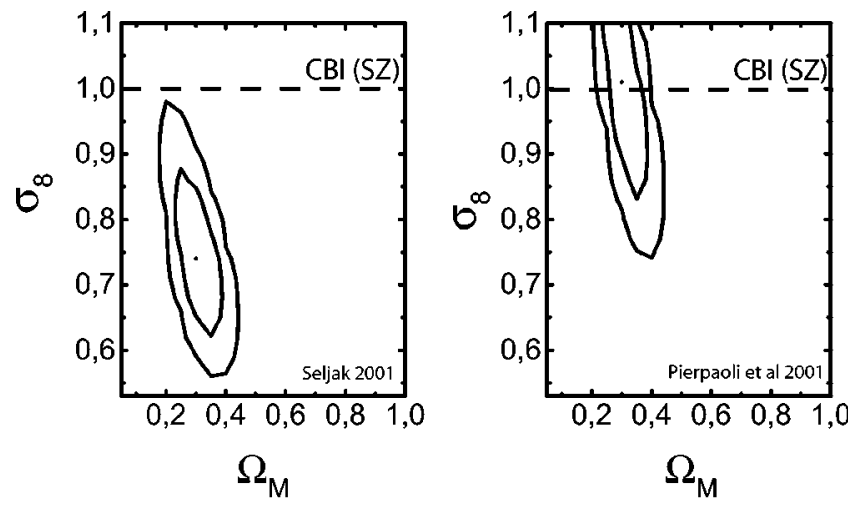

these small scales such as those from the kinetic SZ effect, the Ostriker-Vishniac effect [27], and from the patchy, or inhomogeneous, reionization [28], are generally expected to be much lower than the thermal SZ contribution. ${ }^{1}$

\section{BEYOND SZ: CONTRIBUTIONS TO SMALL SCALE POWER}

In addition to such processes, the anisotropies at small angular scales can also be affected by the presence of foregrounds. At frequencies relevant to recent small-scale anisotropy results at $30 \mathrm{GHz}$, a dominant contribution may come from extra-galactic radio point sources. The possible contamination involving radio point sources has been investigated in [6] with the conclusion that the contribution from point sources should not be significant. It has been recently noted [31], however, that if the excess power at small angular scales is to be explained by the $\mathrm{SZ}$ effect, then radio point sources at the observing frequencies of CBI and BIMA, 30 $\mathrm{GHz}$, must be a surprisingly weak contaminant.

In this paper we want to point out that an extra contribution to temperature anisotropies at small angular scales can arise also in nonstandard models of inflation. A strong possibility, for example, is the one that has recently been discussed in detail [32] involving the existence of primordial voids in the early universe ${ }^{2}[32-34]$. There is an important contribution from these voids to temperature anisotropies. Similar to the Sachs-Wolfe (SW) ([35]) effect associated with the dark matter potential at the last scattering surface, these primordial voids also generate a SW contribution. The angular scale for this latter SW temperature fluctuation is consistent with the projected size of the void at the last scattering surface. Note that voids which are fully embedded in the primordial photon-baryon fluid do not generate a new anisotropy contribution via the SW effect.

Following the discussion presented in Refs. [34] and [32], for illustration purposes, we calculate the SW contribution associated with voids using parameters which are consistent with voids observed via redshift surveys of the present day and a volume fraction, again, consistent with such observations [36]. Between the last scattering surface and today, these voids also contribute to additional temperature anisotropies through frequency shifts, mainly the ReesSciama (RS) effect [37] and effects such as gravitational lensing. For parameters on voids consistent with current observations, these latter low redshift contributions are, however, smaller and can be ignored [34].

Another possibility, recently investigated by various authors (see e.g. [38]) but in different contexts, is the presence of a feature in the primordial spectrum of fluctuations, as expected, for example, in inflationary models with broken

ments can be strongly affected by systematics, it appears that the cosmological information obtained from the secondary anisotropies under the assumption of a pure SZ component is in tension with the same information obtained by the primary anisotropies when combined with other cosmological observables.

A solution to this problem, however, is to postulate an extra contribution to the small-scale anisotropies from some mechanism different from SZ. Additional contributions at

\footnotetext{
${ }^{1}$ We refer the reader to Refs. [29] and [30] for a detailed discussion of these contributions.

${ }^{2}$ For the purpose of this discussion, note that we define the early universe to be the era around, and before, recombination $(z$ $\sim 1000)$, while the local universe is the era after reionization $(z$ $\lesssim 10)$.
} 

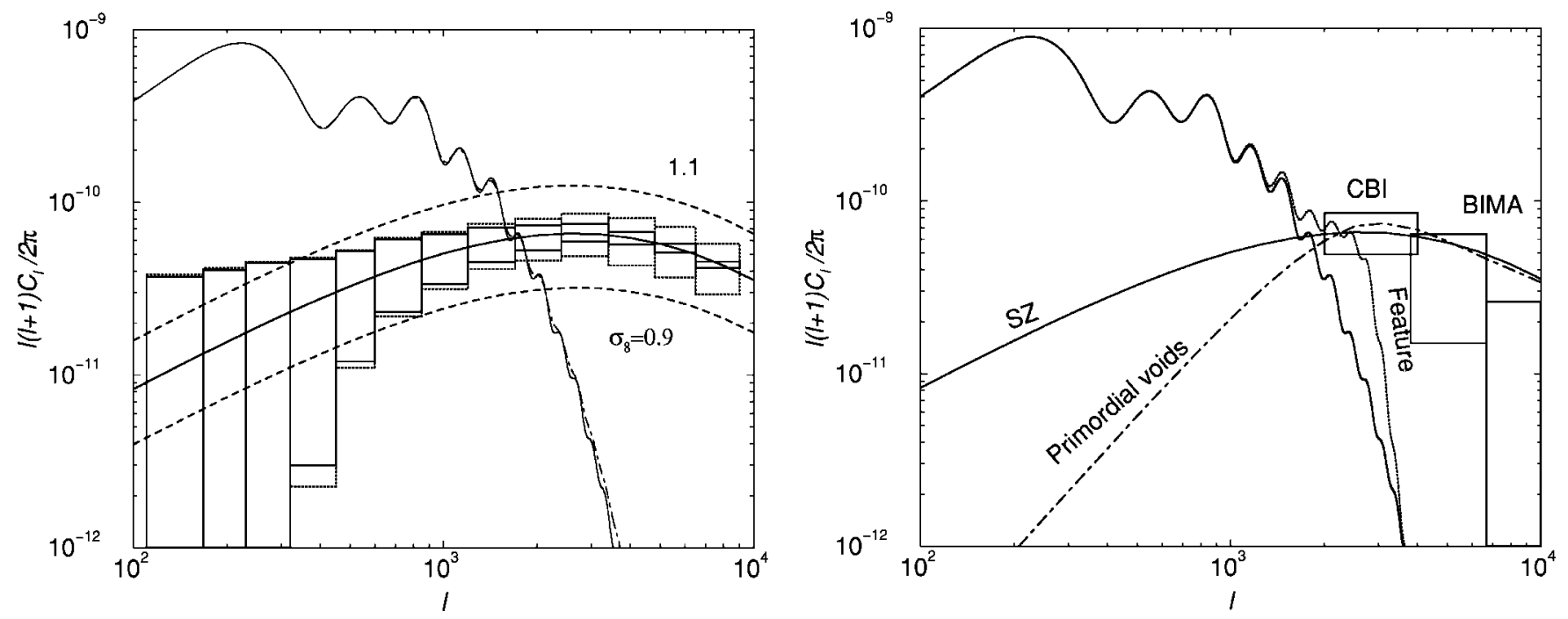

FIG. 2. Left: The thermal SZ power spectrum. The three curves show the variation in the SZ contribution due to a change in the normalization about $\sigma_{8}=1.0$. Due to the highly nonlinear behavior, the SZ thermal contribution is strongly dependent on the normalization of the matter power spectrum. The two sets of error bars show the highly non-Gaussian behavior of the clusters that contribute: the small error bars in solid are the ones expected under a Gaussian description, while the dotted errors bars show the total errors including the covariance due to non-Gaussianities. For illustration, we have assumed a no instrumental noise survey of 1 sqr. degrees. In addition to increasing the errors by a factor of a few, non-Gaussianities also correlate the arcminute scale band power estimates at the $50 \%$ to $90 \%$ level. Right: Possible contributions to small scale power from effects related to the early universe. These include a contribution from a feature in the primordial spectrum of fluctuations (dotted line), and a distribution of primordial voids at the last scattering surface (dot-dashed line). For comparison, we also show the SZ contribution and the standard prediction for the anisotropy power spectrum.

scale invariance. In Fig. 1, we present the possible contribution to the small-scale anisotropies from a feature in the spectrum modeled as Gaussian-centered at $k$ $\sim 0.20\left(\mathrm{~h} \mathrm{Mpc}^{-1}\right)$ with dispersion $\Delta k \sim 0.03\left(\mathrm{~h} \mathrm{Mpc}^{-1}\right)$ and amplitude $A \sim 5$. While this feature is not fully ruled out by results on the matter power spectrum from recent galaxy redshift surveys such as the $2 \mathrm{dF}$ (see e.g. [39]), its width is still dangerously close to the observed spectral resolution around these scales. Though models involving primordial features only produce excess power over a limited range in multipolar space, in this case only out to $l \sim 3000$, the current smallscale anisotropy observations are also limited in the coverage of the power spectrum, complicating any identification of a feature. However, as we can see from Fig. 2, the BIMA result is already strongly constraining this hypothesis.

In addition to all these possible contributions arising from modifications to the standard inflationary scenario, we also note that there may be additional possibilities to generate small-scale anisotropies at the last scattering via modifications to recombination [40].

Given distinct possibilities for small-scale temperature anisotropies, involving the large-scale structure after reionization and the last scattering surface, and given the difficulties in explaining the CBI effect as solely due to SZ, an important question is how to distinguish between them when interpreting any detection of power at smaller angular scales in current and future experiments.

In the rest of the discussion, we will therefore consider how likely it is that we can distinguish between the two scenarios involving an early universe contribution or a local redshift contribution. For the purpose of this discussion, we will assume potential low redshift contributions involving SZ and radio point sources.

\section{EARLY UNIVERSE OR LOCAL STRUCTURES?}

Note that a fundamental aspect related to the SZ contribution is its distinct frequency dependence. This spectrum can be utilized to separate its contribution from the dominant anisotropies associated with primary fluctuations at large scale and other thermal fluctuations, such as due to the SZ kinetic effects associated with the peculiar motions of clusters, as well as any void or nonstandard contribution, at small angular scales [41]. The current anisotropy observations at small scales, unfortunately, are limited at most to a single frequency and this limitation is unlikely to be improved significantly until the advent of the next generation of experiments. In the case of CBI and BIMA, observations are limited to $30 \mathrm{GHz}$ at the RJ part of the spectrum. We expect observations at higher frequencies, such as at $150 \mathrm{GHz}$ by the ACBAR instrument, to see a contribution which is lower than at $30 \mathrm{GHz}$, by a factor of $\sim 0.22$. Observations at and above the SZ null frequency of $217 \mathrm{GHz}$ are clearly desirable since a contribution from radio point sources is also expected to be decreasing in frequency while an early-universe contribution is frequency-independent.

An additional aspect of the SZ effect is its nonGaussianity. It is now well established that contributions to the SZ effect primarily come from massive galaxy clusters, which are rare. Such a mass dependence makes the SZ effect highly nonlinear. As illustrated in Fig. 2, for example, the SZ effect varies by a factor of $\sim 2$ when the normalization of the 
matter power spectrum is changed by $\sim 10 \%$. Another aspect of this nonlinearity is the increase in SZ variance when compared to the expected Gaussian variance contribution. In addition to SZ, point source contribution may also be nonGaussian, especially if the point sources trace the nonlinear large-scale structure at low redshifts. Thus, non-Gaussian aspects are potentially common to all low redshift contributions since they all tend to trace the local universe nonlinear structures which are non-Gaussian distributed.

The non-Gaussianity alone, however, may not distinguish the nature of small-scale anisotropy power as observed in current experiments, as the nonstandard modifications to the last scattering involving primordial voids also generate a highly non-Gaussian anisotropy contribution [42]. A reliable approach to distinguish between a local contribution, whether it be SZ or point sources, and an early contribution, such as voids and bumps in the primordial power spectrum, is to consider a combined study involving the large-scale structure and CMB. Here, we suggest a cross correlation of the CMB anisotropy data with a map of the large-scale structure.

The correlation between CMB, mainly the best Cosmic Microwave Background (COBE) Differential Microwave Radiometer (DMR) map, and large scale structure has already been considered to explain the extent to which the integrated SW (ISW) effect contributes at large angular scales [43]. As discussed in [44], this correlation, however, is dominated by the large cosmic variance at low multipoles corresponding to angular scales with tens of degrees on the sky. In the case of small-scale anisotropies, the extent to which the correlation can be detected will be determined primarily by the instrumental noise contribution. For the pur-

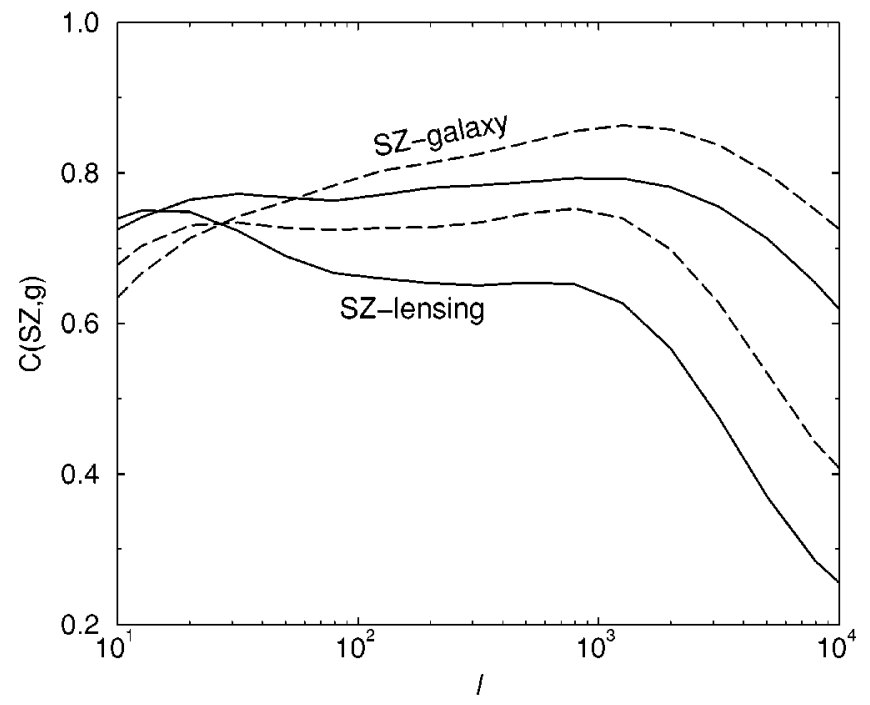

pose of this discussion, we introduce the correlation coefficient between, say, large-scale structure contributions (SZ or point sources) and a tracer of the large-scale structure as

$$
\operatorname{Corr}(\mathrm{LSS}, i)_{l}=\frac{C_{l}^{\mathrm{LSS}-i}}{\sqrt{C_{l}^{\mathrm{LSS} C_{l}^{i}}}},
$$

where $C_{l}^{\mathrm{LSS}-i}$ is the cross power spectrum between largescale structure contributions and the tracer field. We write this as $C_{l}^{\mathrm{LSS}-i}=C_{l}^{\mathrm{SZ}-i}+C_{l}^{\mathrm{PS}-i}$, where $C_{l}^{\mathrm{SZ}-i}$ and $C_{l}^{\mathrm{PS}-i}$ are the cross power spectra between SZ and the tracer field, and point sources (PS) and the tracer field, respectively. The cross correlation of SZ and the tracer field is described in Ref. [13] following the so-called halo model approach to large-scale structure [45]; we note that a similar approach can be considered for $C_{l}^{\mathrm{PS}-i}$. Given the poor knowledge of the statistical distribution of radio-galaxies, which could represent a different population from those observed in the optical distribution, and the wide range of observed spectral energy distributions due to different synchrotron cutoffs, however, the problem of predicting radio source clustering properties in a given band, as well as their correlation with other LSS tracers, is a difficult, though interesting, task. We note that certain issues related to point sources are discussed in [31], to which we refer the reader for further details.

Note that the associated signal-to-noise ratio for the detection of the cross correlation is

$$
\left(\frac{\mathrm{S}}{\mathrm{N}}\right)^{2}=f_{\mathrm{sky}} \sum_{l} \frac{(2 l+1) \operatorname{Corr}^{2}(\mathrm{LSS}, i)_{l}}{\operatorname{Corr}^{2}(\mathrm{LSS}, i)_{l}+\left(1+\frac{N_{l}^{\mathrm{LSS}}}{C^{\mathrm{LSS}}}\right)\left(1+\frac{N_{l}^{i}}{C_{l}^{i}}\right)},
$$

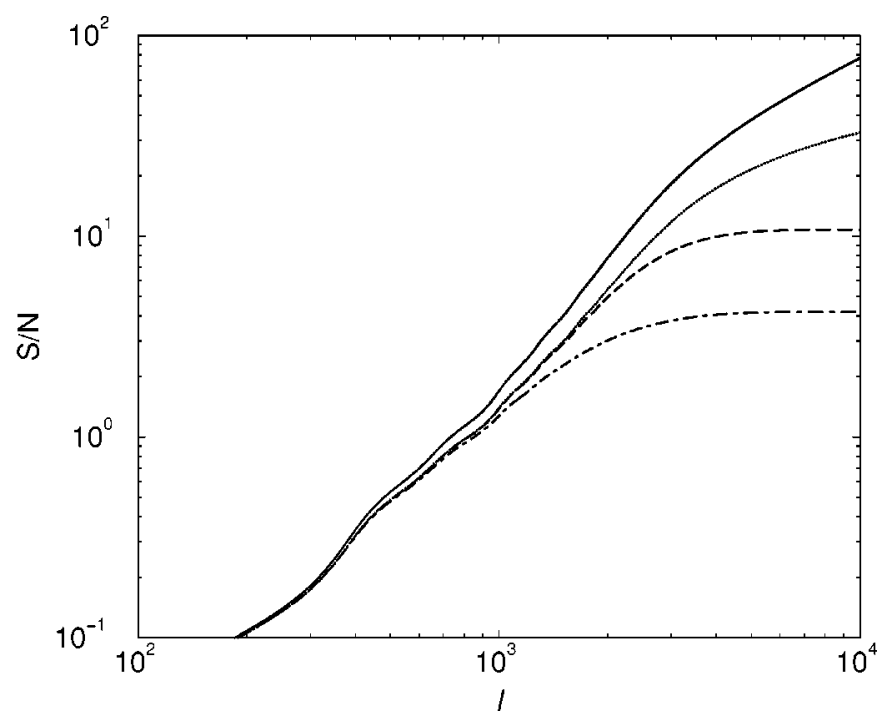

FIG. 3. Left: The SZ large-scale structure correlation coefficients. The curves are for large-scale structure tracers involving galaxies, with a median redshift of 0.5 (dashed bottom) and 1.0 (dashed top) and weak lensing, with background sources at redshifts of 1.0 (solid bottom) and 3.0 (solid top). In general, large-scale structure tracers are correlated with the SZ effect at arcminute scales with correlation coefficients of order $\sim 0.6$, when the median redshift involved is of order 1. Right: The cumulative signal-to-noise ratio for the detection of SZ-galaxy cross correlation. We assume a survey of 10 degrees $^{2}$ and a year of observations. The solid line is the maximum with no instrumental noise or shot-noise contribution, while the dotted line is the signal-to-noise ratio with a galaxy surface density of $10^{9} \mathrm{sr}^{-1}$, the dashed line is the signal-to-noise ratio with an additional instrumental noise for the small-scale CMB experiment with a sensitivity of $25 \mu \mathrm{K} \sqrt{\mathrm{sec}}$ and a beam of 2 arcmins (FWHM), and the dot-dashed line is the ratio when the sensitivity is $100 \mu \mathrm{K} \sqrt{\mathrm{sec}}$. 
where $\quad C^{\mathrm{LSS}}=C_{l}^{\mathrm{SZ}}+C_{l}^{\mathrm{PS}}+C_{l}^{\mathrm{SZ}-\mathrm{PS}}, \quad N_{l}^{\mathrm{LSS}}\left(=C_{l}^{\mathrm{CMB}}+C_{l}^{\mathrm{LSS}}\right.$ $+C_{l}^{\text {noise }}$ ) and $N_{l}^{i}$ are the total power spectrum contributions from large-scale structure noise contributions associated with small-scale fluctuations and the power spectrum of the $i$ tracer field, respectively. Here, $C_{l}^{\text {SZ-PS }}$ is the cross-power spectrum between the SZ effect and contaminant point sources; the latter can again be calculated following the models of Ref. [29]. If the $i$ th field is galaxies (which may also contribute to the radio background), then we note that $C_{l}^{\mathrm{SZ}-\mathrm{i}}$ $\propto C_{l}^{\mathrm{SZ}-\mathrm{PS}}$. Note that at small scales, $C_{l}^{\mathrm{CMB}} \ll C_{l}^{\mathrm{LSS}}$.

In Fig. 3, we show the correlation coefficient of SZ and tracers of the large-scale structure involving weak lensing convergence and the galaxy distribution. In the near future, a cross-correlation study of small-scale anisotropies and a map of the galaxy distribution, such as from the Sloan survey, looks promising and should be considered. To calculate the expected signal-to-noise ratio for a detection of the correlation signal, we assume a noise contribution to the tracer field involving the shot-noise contribution arising from the finite number of galaxies and assume no point source contribution to the small-scale anisotropy signal. The latter is a safe assumption given that point source contributions have been found to be subdominant by various monitoring and analysis techniques considered by, at least, the CBI group. For a survey of 10 degrees $^{2}$ and no noise contributions, we estimate the signal-to-noise ratio of order $\sim 100$ while this drops to $\sim 10$ when reasonable noise contributions are considered in both the temperature and galaxy tracer field. For example, the galaxy shot noise considered here involves a surface density of $10^{9} \mathrm{sr}^{-1}$, which is the density of galaxies down to an $R$ band magnitude of 25 [46]. We note that the presence of point sources will likely increase this signal-to-noise estimate considerably since point sources may be correlated with tracers of the large-scale structure, such as the galaxy distribution field. This increase clearly motivates attempts for such a combined study.

Thus, the extent to which small-scale anisotropies correlate with large-scale structure by itself does not determine the nature of the temperature fluctuation. Though it estab- lishes that the local universe is partly responsible, it does not necessarily mean that SZ is the dominant contribution since fluctuations may contain a point source contribution. If one is interested in distinguishing between SZ and point sources, it is necessary to consider aspects beyond simple cross correlations.

In general, the extent to which the SZ thermal effect contributes at small angular scales can be established more reliably based on its spectral dependence relative to thermal CMB than any correlation associated with the large-scale structure alone. The SZ frequency spectrum is unique and is unlikely to be mimicked by other sources of contributions. To determine the SZ contribution and to separate it from other thermal fluctuations at small angular scales, the future experiments should be equipped with multifrequency capabilities that expand from low RJ frequencies to $\sim 300 \mathrm{GHz}$; the low frequencies help determine the radio source contribution while high frequencies determine the confusion from mid infrared/submm point sources. The observations at SZ null of $217 \mathrm{GHz}$ determine the extent to which other thermal fluctuations are significant as a source of small-scale anisotropies.

Though to a certain extent current and upcoming detection of small-scale power may be important as a first detection, dedicated small angular scale experiments with multifrequency coverage are clearly needed to fully understand the nature of fluctuations at these small scales. Just as anisotropy studies at degree angular scales involving the acoustic peak structure have been successful as a strong probe of cosmology, the small-scale anisotropies open the window to understand both the large scale structure and important subtle effects involving the last scattering surface [47-49].

\section{ACKNOWLEDGMENTS}

We wish to thank Martin Kunz, Louise Griffiths, and Joe Silk for helpful comments. This work was supported at Caltech by the Sherman Fairchild Foundation and DOE Grant No. DE-FG03-92-ER40701. A.M. is supported by PPARC.
[1] Boomerang Collaboration, C.B. Netterfield et al., Astrophys. J. 571, 604 (2002).

[2] N.W. Halverson et al., Astrophys. J. 568, 38 (2002).

[3] A.T. Lee et al., Astrophys. J. Lett. 561, L1 (2001).

[4] P.F. Scott et al., astro-ph/0205380.

[5] T.J. Pearson et al., astro-ph/0205388.

[6] B.S. Mason et al., astro-ph/0205384.

[7] K.S. Dawson et al., astro-ph/0206012.

[8] J.R. Bond et al., astro-ph/0205386.

[9] E. Komatsu and U. Seljak, astro-ph/0205468.

[10] R.A. Sunyaev and Ya.B. Zel'dovich, Mon. Not. R. Astron. Soc. 190, 413 (1980).

[11] J.E. Carlstrom, M. Joy, and L. Grego, Astrophys. J. Lett. 456, L75 (1996); M. Jones, R. Saunders, and P. Alexander et al., Nature (London) 365, 320 (1993).

[12] M. White, L. Hernquist, and V. Springel, astro-ph/0205437.
[13] A. Cooray, Phys. Rev. D 62, 103506 (2000).

[14] A. Cooray, Phys. Rev. D 64, 063514 (2001).

[15] Y.T. Lin and J.J. Mohr, astro-ph/0208005.

[16] A. Refregier et al., astro-ph/0203131.

[17] E. Pierpaoli et al., Mon. Not. R. Astron. Soc. 325, 77 (2001).

[18] P. Viana et al., astro-ph/0111394.

[19] U. Seljak, astro-ph/0111362.

[20] N. Bahcall et al., astro-ph/0205490.

[21] J.L. Sievers, astro-ph/0205387.

[22] P. Schuecker et al. (in preparation); see also L. Guzzo, astro-ph/0207285.

[23] O. Lahav et al., astro-ph/0112162; Mon. Not. R. Astron. Soc. (to be published).

[24] A. Melchiorri and J. Silk, Phys. Rev. D 66, 041301(R) (2002).

[25] N. Gnedin, astro-ph/0110290.

[26] M. Bruscoli, A. Ferrara, and E. Scannapieco, 
astro-ph/0201094.

[27] J.P. Ostriker and E.T. Vishniac, Astrophys. J. Lett. 306, L51 (1986).

[28] N. Aghanim, F.X. Desert, J.L. Puget, and R. Gispert, Astron. Astrophys. 311, 1 (1996).

[29] A. Cooray, astro-ph/0203048.

[30] N. Aghanim, P.G. Castro, A. Melchiorri, and J. Silk, astro-ph/0203112.

[31] G.P. Holder, astro-ph/0205467.

[32] L.M. Griffiths, M. Kunz, and J. Silk, astro-ph/0204100.

[33] L. Amendola, C. Baccigalupi, and F. Occhionero, Astrophys. J. Lett. 492, L5 (1998).

[34] N. Sakai, N. Sugiyama, and J. Yokoyama, Astrophys. J. 510, 1 (1999).

[35] R.K. Sachs and A.M. Wolfe, Astrophys. J. 147, 73 (1967).

[36] F. Hoyle and M.S. Vogley, Astrophys. J. 566, 641 (2002).

[37] M.J. Rees and D.N. Sciama, Nature (London) 519, 611 (1968).

[38] O. Elgaroy et al., Phys. Rev. Lett. 89, 061301 (2002); J. Barriga, E. Gaztanaga, M.G. Santos, and S. Sarkar, Mon. Not. R.
Astron. Soc. 324, 977 (2001); astro-ph/0010571.

[39] M. Tegmark, A.J.S. Hamilton, and Y. Xu, astro-ph/0111575.

[40] M. White, Astrophys. J. 555, 88 (2001).

[41] A. Cooray, W. Hu, and M. Tegmark, Astrophys. J. 540, 1 (2000).

[42] A. Cooray et al. (in preparation).

[43] S. Boughn, R. Crittenden, and N. Turok, New Astron. 3, 275 (1998); A. Cooray, Phys. Rev. D 65, 103510 (2002).

[44] Cooray [43].

[45] A. Cooray and R. Sheth, Phys. Rep. (to be published), astro-ph/0206508.

[46] I. Smail, D. Hogg, Y. Lin, and J.G. Cohen, Astrophys. J. Lett. 449, L105 (1995).

[47] S. Borgani, astro-ph/0106428.

[48] R. Bean and A. Melchiorri, Phys. Rev. D 65, 041302(R) (2002).

[49] U. Seljak, Astrophys. J. 463, 1 (1996); W. Hu, Phys. Rev. D 62, 043007 (2000). 